hep-ph/9506362

Astropart.Phys.(to appear)

\title{
Astrophysical and cosmological considerations on a string dilaton of a least coupling
}

\author{
C. E. Vayonakis \\ Physics Department, University of Ioannina, \\ GR-451 10 Ioannina, Greece円 \\ and \\ Physics and Astronomy Subject Group, \\ University of Sussex, Brighton BN1 9QH, U.K.
}

\begin{abstract}
String-loop effects may generate very weak matter couplings for a (massless) dilaton. We examine limits on the shift of such a dilaton toward its present equilibrium value from bigbang nucleosynthesis and the binary pulsar. On the other hand, the approach of the dilaton toward its present value can be realized very early in the universe in a quick and efficient way if an inflationary period is present. We comment briefly on some implications.
\end{abstract}

\footnotetext{
${ }^{1}$ Permanent address
} 


\section{Introduction}

String theory provides, at present, the only scheme capable to give a promising unified picture of gauge particle interactions and quantum gravity. Within this theory, there is the usual tensor Einstein graviton always intimately combined with a universal partner: the dilaton. The mass of the dilaton is presently unkown. Its couplings to matter a priori generate deviations from Einstein gravity. In fact, short distance tests of Newtonian gravity can be used to put bounds on the dilaton mass. Based on Eötvös-type experiments and satellite observations one can place a bound $m_{\phi} \gtrsim 10^{-4} \mathrm{eV}$ [1]. On the other hand, Cavendishtype experiments, which probe deviations from the inverse-square law, can be used to put a lower bound on the mass of a tree-level string dilaton $m_{\phi} \gtrsim 10^{-3} \mathrm{eV}$ [2]. Present conventional wisdom is to have a non-vanishing dilaton mass [3], possibly connected with a (dynamical) supersymmetry breaking mechanism [4].

A very interesting proposal, however, has been put forward in ref. [2] (see also [5]), offering the alternative possibility of a massless dilaton. In this, it is pointed out that nonperturbative string-loop effects (associated with worldsheets of arbitrary genus in indermediate string states) may naturally reconcile the existence of a massless dilaton with experimental data, if the various couplings of the dilaton field to the other fields exhibit a certain kind of universality (as the tree-level dilaton couplings do). Then, under the assumption that the different coupling functions $B_{i}(\Phi)$ have extrema at some point $\Phi=\Phi_{m}$, the study of the cosmological evolution of a general graviton-dilaton-matter system during the radiation- and the matter -dominated eras shows [2] that the dilaton is cosmologically attracted toward the value $\Phi_{m}$, where it decouples from matter. This illustrates a "least coupling" mechanism in the sense that the universe is attracted to dilaton values extremizing the strengths of its interactions. The attaction mechanisms discussed in [2] (see also [6]), driving the vacuum expectation value of the dilaton toward values at which it decouples from matter, are not however perfect attractors. It remains, then, the important question of giving quantitative independent estimates of these residual coupling strengths of the dilaton.

\section{The relaxation mechanism paradigm}

To be concrete, let us summarize in this section the proposal of ref. [2] by considering the 
effective action for the string massless modes

$$
S=\int d^{4} x \sqrt{\hat{g}} B(\Phi)\left\{\frac{1}{\alpha^{\prime}} \hat{R}+\frac{1}{\alpha^{\prime}}\left[4 \hat{\nabla}^{2} \Phi-4(\hat{\nabla} \Phi)^{2}\right]-\frac{k}{4} \hat{F}^{2}-\overline{\hat{\psi}} \hat{\nabla} \hat{\psi}-\frac{1}{2}(\hat{\nabla} \hat{\sigma})^{2}-\hat{V}(\hat{\sigma})\right\}
$$

where the dilaton coupling function $B(\Phi)$ appearing as a common factor in front admits a series expansion of the form

$$
B(\Phi)=e^{-2 \Phi}+c_{0}+c_{1} e^{2 \Phi}+c_{2} e^{4 \Phi}+\ldots
$$

The first term in the expansion (2) is the string tree-level contribution (corresponding to spherical topology for indermediate worldsheets), which is known to couple the dilaton in a universal multiplicative manner to all other fields and is derived from the fact that $g_{s}=\exp (\Phi)$ plays the role of the string coupling constant [7]. The remaining terms represent the string-loop effects: the genus-n string-loop contributions to any string transition amplitude contains a factor $g_{s}^{2(n-1)}=\exp [2(n-1) \Phi]$. At the present stage of development of string theory, little is known about the global behaviour of dilaton coupling function $B(\Phi)$, apart from the fact that in the limit $\Phi \rightarrow-\infty\left(g_{s} \rightarrow 0\right)$ it should admit a series expansion in powers of $g_{s}^{2}=\exp [2 \Phi]$. In general, one expects to have various dilaton coupling functions $B_{i}(\Phi)$ as coefficients in front of each term appearing in the action. For the cosmological attraction mechanism discussed in [2] to work, it suffices that a universality condition must be fulfilled, namely that the various coupling functions $B_{i}(\Phi)$ must all admit a local maximum at some common value $\Phi_{m}$. For the action (1) this universality is guaranteed by the factorization of the common function $B(\Phi)$. In that case, string-loop effects as in (2) can allow this function to admit a local maximum. In the following we assume such a common function $B(\Phi)$. It might be stressed that the desired universality could be attributed to the conjectured S-duality of the string theory [8], namely the minimum/maximum (weak/strong) coupling constant duality $g_{s} \rightarrow \frac{1}{g_{s}}$ corresponding to the discrete symmetry $\Phi \rightarrow-\Phi$. This property is intrinsically non-perturbative and would ensure that all physical quantities have extremum at $\Phi_{m}=0$. A similar duality symmetry is known to hold for the other gaugeneutral (massless) scalar fields present in string theory, namely those associated with the compactified dimensions and known as moduli. The latter symmetry, called T-duality or 
target-space duality [9] ( a minimum/maximum length duality $R \rightarrow \frac{\alpha^{\prime}}{R}$ ) is manifest order by order in string perturbation theory (though non-perturbative on the string worldsheet). It is true that, unlike T-duality, S-duality has not yet been proved to be a string theory symmetry, although some interesting and non-trivial tests can be carried out 10. There is, in any case, some evidence coming from different directions that S- and T-duality are part of a larger unified symmetry structure [11]. Under the spirit of these considerations, it was conjectured [2, 5] that the proposed attractor mechanism could be invariably applied to the dilaton as well as the various moduli fields.

It is convenient to transform the action (1) by introducing several $\Phi$-dependence rescalings. In particular, one can put the gravity sector (graviton, dilaton) and the matter one (fermions, gauge fields, scalars) into a standard form by replacing the original "string frame" metric $\hat{g}_{\mu \nu}$ by a conformally related "Einstein frame" metric

$$
g_{\mu \nu}=C B(\Phi) \hat{g}_{\mu \nu}
$$

the original field $\Phi$ by a canonical scalar field

$$
\phi=\int d \Phi\left[\frac{3}{4}\left(\frac{B^{\prime}}{B}\right)^{2}+2 \frac{B^{\prime}}{B}+2\right]^{1 / 2}
$$

( where prime denotes $\mathrm{d} / \mathrm{d} \Phi$ ) and the original Dirac field $\hat{\psi}$ and scalar field $\hat{\sigma}$ by

$$
\psi=C^{-3 / 4} B^{-1 / 4} \hat{\psi}, \sigma=C^{-1 / 2} \hat{\sigma}
$$

The transformed action reads

$$
\begin{gathered}
S=S_{g r}(g, \phi)+S_{m}(\psi, A, \sigma, \ldots) \\
S_{g r}=\int d^{4} x \sqrt{g}\left[\frac{1}{4 q} R-\frac{1}{2 q}(\nabla \phi)^{2}\right] \\
S_{m}=\int d^{4} x \sqrt{g}\left[-\bar{\psi} \nabla \psi-\frac{k}{4} B(\phi) F^{2}-\frac{1}{2}(\nabla \sigma)^{2}-B^{-1}(\phi) V(\sigma)+\ldots\right]
\end{gathered}
$$

where $q=4 \pi \bar{G}=\frac{1}{4} C \alpha^{\prime}$ with $\bar{G}$ denoting a bare gravitational coupling constant and $B(\phi) \equiv$ $B[\Phi(\phi)]$. The constant $\mathrm{C}$ can be chosen so that the string units and the Einstein units 
coincide at the present cosmological epoch $C B\left(\Phi_{0}\right)=1$. The relation of string models to the observed low-energy world is through the dilaton dependence (from (6.c)) of the gauge coupling constants

$$
g^{-2}(\phi)=k B(\phi)
$$

Furthermore, one still needs to take into account the quantum effects of the light modes between the string scale in Einstein units

$$
\Lambda_{s}(\phi)=C^{-1 / 2} B^{-1 / 2}(\phi) \hat{\Lambda_{s}}
$$

and some (low) observational scale. In eq.(8) $\hat{\Lambda}_{s} \simeq 3 \times 10^{17} \mathrm{GeV}$ [12] is the (string frame) string unification scale $\sim\left(a^{\prime}\right)^{-1 / 2}$. So, for example, the renormalization group implies that, at the one-loop level, the QCD mass scale $\Lambda_{Q C D}$ is exponentially related to the inverse of $g_{3}$ gauge coupling constant and through the above dependense we have

$$
\Lambda_{Q C D}(\phi) \sim \Lambda_{s}(\phi) \exp \left(-8 \pi^{2} b_{3}{ }^{-1} g_{3}{ }^{-2}\right)=C^{-1 / 2} B^{-1 / 2}(\phi) \exp \left[-8 \pi^{2} b_{3}{ }^{-1} k_{3} B(\phi)\right] \hat{\Lambda}_{s}
$$

Later on we will need the dilaton dependence of the ( Einstein frame) QCD part of the mass of the nucleons, which is given by some pure number times $\Lambda_{Q C D}(\phi)$. Furthermore, the mass ( in Einstein units) of any type of particle, labelled A, will depend on $\phi$ only through the function $B(\phi)$

$$
m_{A}(\phi)=m_{A}[B(\phi)]
$$

We assume a form dependence as suggested by (9), that is

$$
m_{A}(\phi)=\mu_{A} B^{-1 / 2}(\phi) \exp \left[-8 \pi^{2} \nu_{A} B(\phi)\right] \hat{\Lambda}_{s}
$$

with $\mu_{A}, \nu_{A}$ pure numbers $\sim O(1)$. This is in fact the case in models in which the electroweak gauge symmetry is broken radiatively, more specifically in the no-scale supergravity models [13.

Upon studying the cosmological evolution of the graviton-dilaton-matter system,the authors of ref. [2] find that the dilaton vacuum expectation value $\phi$ is dynamically driven toward some finite value $\phi_{m}$, where the mass functions $m_{A}(\phi)$ reach a local minimum corresponding 
to a local maximum of $B(\phi)$. The important quantities in the proposal under discussion are the attraction shift $\Delta \phi \equiv\left(\phi-\phi_{m}\right)$ of the $\phi$ toward the value $\phi_{m}$ during the evolutionary history of the universe and the coupling strength

$$
\alpha_{A}(\phi)=\frac{\partial}{\partial \phi} \ln m_{A}(\phi)
$$

of the dilaton to the A-type particles. This is seen from the field equation for $\phi$, which is

$$
\nabla^{2} \phi=-q \Sigma
$$

where the source term is

$$
\Sigma=g^{-1 / 2} \frac{\partial}{\partial \phi} S_{m}
$$

Upon writing for the material content of the universe the action

$$
S_{m}=-\sum_{A} \int m_{A}(\phi) d \tau_{A}
$$

we have

$$
\Sigma=\sum_{A} \alpha_{A}(\phi) T_{A}
$$

where $T_{A}=-\rho_{A}+3 p_{A}$ is the trace of the $A$-type contribution to the total energy-momentum tensor $T^{\mu} \nu=\sum_{A} T_{A}{ }^{\mu} \nu$. Thus, $\alpha_{A}(\phi)$ is the coupling strength of the dilaton to the A-type particles and its square $\alpha_{A}^{2}(\phi)$ will appear in all quantities involving interactions mediated by exchanges of dilatons ( in the same way as $g^{2}$ appears in all gauge interactions).

The main parameter determining both the efficiency of the cosmological relaxation of $\phi$ toward $\phi_{m}$ and the coupling strength $\alpha_{A}(\phi)$ is the curvature $\kappa$ of the function $\ln B(\phi)$ near its maximum at $\phi_{m}$. In the quadratic approximation we have

$$
\ln B(\phi) \simeq \text { const. }-\frac{1}{2} \kappa\left(\phi-\phi_{m}\right)^{2}
$$

Then, from (11) and (12) one gets

$$
\alpha_{A}(\phi) \simeq \beta_{A}\left(\phi-\phi_{m}\right)
$$


with $\beta_{A}=\kappa \ln e^{1 / 2} \hat{\Lambda}_{s} / m_{A} \simeq 40 \kappa(\kappa \sim O(1))$.

As discussed by the authors of ref. [2], because of the steep dependence of $m_{A}(\phi)$ on $B(\phi)$ [see eq.(11)], during the radiation-dominated era each time the cosmic temperature $\mathrm{T}$ becomes of the order of the mass threshold $m_{A}$ of some particle, $\phi$ is attracted towards $\phi_{m}$ by a factor $\sim 1 / 3$. Besides mass thresholds, the electroweak and the QCD phase transitions provide other possible attractor processes during the same period. During the subsequent matter-dominated era, $\phi$ is further attracted toward $\phi_{m}$ by a factor roughly proportional to $Z_{0}^{-3 / 4}$, where $Z_{0} \simeq 1.3 \times 10^{4}$ is the redshift seperating us from the end of the radiation era. All this leads to a present value $\phi_{0}$ near but different from $\phi_{m}$ by a very small amount $\Delta \phi_{0}=\left(\phi_{0}-\phi_{m}\right)$ and to a cosmologically relaxed dilaton coupling to matter around us with a strength

$$
\alpha_{A}\left(\phi_{0}\right)=\left.\frac{\partial}{\partial \phi} \operatorname{lnm}_{A}(\phi)\right|_{\phi=\phi_{0}} \simeq \beta_{A}\left(\phi_{0}-\phi_{m}\right)
$$

¿From the evolution of the dilaton during the two well-separated eras of classical cosmology, the authors of ref. [2] conclude that today $\left(\phi_{0}-\phi_{m}\right) \simeq 10^{-9}$. Such a very small amount implies, of course, very small deviations from general relativity. In particular, all deviations from Einstein's theory ( including post-Newtonian deviations measured by the two Eddington parameters $1-\gamma_{E d d}$ and $\beta_{E d d}-1$, residual cosmological variation of the coupling constants and violation of the weak equivalence principle) are proportional to a small factor $\left(\phi_{0}-\phi_{m}\right)^{2}$ coming from the exchange of a $\phi$ particle. The previous estimate gives a small factor $\left(\phi_{0}-\right.$ $\left.\phi_{m}\right)^{2} \simeq 10^{-18}$. The present high-precision tests of the equivalence principle reach the $10^{-12}$ level ( corresponding to $\left(\phi_{0}-\phi_{m}\right)_{o b s} \simeq 10^{-6}$ in the context of the present model).

In the next, we are looking for some independent constraints for the relaxation shift $\Delta \phi=\left(\phi-\phi_{m}\right)$ toward its present value $\Delta \phi_{0}=\left(\phi_{0}-\phi_{m}\right)$. We choose two well-established areas of cosmology and astrophysics, namely big-bang nucleosynthesis and binary pulsars. The first offers the possibility to costrain the relaxation shift $\left(\phi-\phi_{m}\right)$ from the time of nucleosynthesis till present, while the second can provide an independent estimate of the present value of $\alpha_{A}\left(\phi_{0}\right) \simeq \beta_{A}\left(\phi_{0}-\phi_{m}\right)$. We will also examine the effect that an inflationary epoch could induce on the relaxation shift under discussion. 


\section{Nucleosynthesis constraint}

Let us first come to nucleosynthesis. Particle physics models are mostly constrained by the mass fraction of primordial ${ }^{4} \mathrm{He}$, usually denoted by $Y_{p}$. The light element abundances including ${ }^{4} \mathrm{He}$ ( i.e. $\mathrm{D},{ }^{3} \mathrm{He},{ }^{4} \mathrm{He},{ }^{7} \mathrm{Li}$ ) are mainly sensitive to the baryon-to-photon ratio $\eta=n_{N} / n_{\gamma}$ within the standard model [14]. Consistency between theory and observations restrict $\eta$ to be in the range $3.1 \times 10^{-10} \lesssim \eta \lesssim 3.9 \times 10^{-10}$. For ${ }^{4} \mathrm{He}$ it is found that [15]

$$
Y_{p}=0.232 \pm 0.003 \pm 0.005
$$

where the uncertainties are $1 \sigma$ statistical and systematic uncertainties, respectively. The ${ }^{4} \mathrm{He}$ abundancy is mainly determined by the neutron-to-proton ratio just before nucleosynthesis

$$
\frac{n}{p} \simeq e^{-\Delta m_{N} / T_{f}}
$$

where $\Delta m_{N}=1.29 \mathrm{MeV}$ is the neutron-proton mass difference and $T_{f} \sim 1 \mathrm{MeV}$ is the freezeout temerature of the weak interactions. Actually, the ratio is slightly altered due to free neutron decays between $T_{f}$ and the onset of nucleosynthesis at about $T \sim 0.1 \mathrm{MeV}$. The temperature $T_{f}$ is determined by the competition between the weak interaction rate and the expansion rate of the universe

$$
G_{F}^{2} T_{f}^{5} \simeq \sqrt{G N} T_{f}^{2}
$$

where $\mathrm{N}$ is the total number of relativistic particle species and $G_{F}, G \simeq \bar{G}$ are Fermi's weak interactions and Newton's gravitational coupling constants, respectively. The ${ }^{4} \mathrm{He}$ abundance is then estimated to be

$$
Y_{p} \simeq \frac{2(n / p)}{1+(n / p)}
$$

Therefore, changes of $Y_{p}$ due to changes in $T_{f}$ and $\Delta m_{N}$ are approximately

$$
\frac{\Delta Y_{p}}{Y_{p}} \simeq\left(\frac{\Delta T_{f}}{T_{f}}-\frac{\Delta\left(\Delta m_{N}\right)}{\Delta m_{N}}\right)
$$

These changes have been discussed in the past in various settings [16] (ours is closer to the last of ref.[16]). In the theory under consideration, the dependence of the various quantities 
of interest on dilaton ( through equations (7)-(11) and (17)) will induce changes on them, which will then constrain the shift $\Delta \phi_{B B N} \equiv\left(\phi-\phi_{m}\right)_{B B N}$ of $\phi$ toward $\phi_{m}$ from the time of big-bang nucleosynthesis to today. Changes in $T_{f}$ are derived from changes in $G_{F}$ and G ( assuming N fixed, see eq.(22)). Since we are working in the "Einstein-frame", we do not consider changes in the gravitational coupling G. Changes in the Fermi coupling $G_{F}$ can be deduced by taking $G_{F}(\phi) \sim m_{A}{ }^{-2}(\phi)$. Since we are only interested in getting the shift of $\phi$ toward $\phi_{m}$ from the time of nucleosynthesis till present, we take that initially $\left(\phi-\phi_{m}\right) \sim O(1)$. We then find

$$
\frac{\Delta T_{f}}{T_{f}} \simeq-50 \Delta \phi_{B B N}
$$

Changes in the neutron-proton mass difference $\Delta m_{N}$, on the other hand, are derived from changes in both the $\Lambda_{Q C D}$-dependent contribution and the fermion (quark) mass contribution to nucleon masses, written as 17

$$
\Delta m_{N} \sim c_{1} \alpha_{e m}(\phi) \Lambda_{Q C D}(\phi)+c_{2} v(\phi)
$$

where $c_{1}, c_{2}$ are dimensionless constants and $v(\phi) \sim G_{F}{ }^{-1 / 2}(\phi)$ is the weak scale vacuum expectation value. Replacing the numerical values of $c_{1}, c_{2}$ [17] and taking the dilaton dependence of the quantities as prescribed within the scheme we consider, we find under the previous assumptions that

$$
\frac{\Delta\left(\Delta m_{N}\right)}{\Delta m_{N}} \simeq 50 \Delta \phi_{B B N}
$$

So, we have a net change in $Y_{p}$

$$
\left|\frac{\Delta Y_{p}}{Y_{p}}\right| \simeq 100 \Delta \phi_{B B N}
$$

¿From the range (20) of values for $Y_{p}$ we see that consistency with big-bang nucleosynthesis requires

$$
\Delta \phi_{B B N} \lesssim 10^{-5}
$$

The derived bound is a kind of "phenomenological" constraint given the successful frame of the big-bang nucleosynthesis and shows the expected shift of the dilaton field from that time up to the present. 


\section{Binary pulsar constraint}

Pulsars, in general [18], and binary pulsars, in particular [19], constitute a unique physics laboratory and can, most probably, provide the ultimate test of gravity theories [20, 21]. The point which is of interest to us here is that the gravitational radiation and the induced change in the period of binary pulsars, most notably the PSR $1913+16$ [22], are so well described within general relativity that any deviations from it are severely constrained.

Different metric theories of gravity predict different possible types ( monopole, higher multipole ) of gravitational radiation emitted by a given source, here the binary pulsar. This can be studied by analysing the effects of gravitational radiation reaction on the source, here the energy loss and the induced change in the period of the binary pulsar. Denote by $m_{1}, m_{2}$ the masses of the pulsar and the companion, $m=m_{1}+m_{2}$ the total mass, $\mu=m_{1} m_{2} / m$ the reduced mass, $\mathrm{r}$ the orbital separation and $\mathrm{v}$ the relative velocity. Then, the rate of energy loss of the system due to the combined effect of quadrupole and monopole gravitational radiation can be written, following ref. [20],

$$
\frac{d E}{d t}=-<\frac{\mu^{2} m^{2}}{r^{4}} \frac{8}{15}\left(k_{1} v^{2}-k_{2} \dot{r}^{2}\right)>
$$

This energy loss induces a decrease in the orbital period $P_{b}$ given by Kepler's third law

$$
\dot{P}_{b} / P_{b}=-\frac{3}{2} \frac{d E}{d t} / E
$$

Carrying out the average over one orbit using Keplerian orbital formulae gives the expressions 20]

$$
\begin{gathered}
\frac{d E}{d t}=-\frac{32}{5}\left(\frac{\mu}{m}\right)^{2}\left(\frac{m}{\alpha}\right)^{5} F(e) \\
\frac{\dot{P}_{b}}{P_{b}}=-\frac{96}{5}\left(\frac{\mu m^{2}}{\alpha^{4}}\right) F(e)
\end{gathered}
$$

where $\alpha$ and e are the orbital semi-major axis and eccentricity, and

$$
F(e)=\frac{1}{2}\left[k_{1}\left(1+\frac{7}{2} e^{2}+\frac{1}{2} e^{4}\right)-k_{2}\left(\frac{1}{2} e^{2}+\frac{1}{8} e^{4}\right)\right]\left(1-e^{2}\right)^{-7 / 2}
$$


Now, within general relativity $k_{1}=12, k_{2}=11$ and formulae (32)-(34) constitute the quadrupole formula for the emission of gravitational energy [23]. In that case, for the parameters of the binary pulsar PSR $1913+16$ formula (33) predicts 20 P $\dot{P}_{b}^{G R}=$ $-(2.40243 \pm 0.00005) \times 10^{-12}$. The observed value is $\dot{P}_{b}^{O B S}=-(2.408 \pm 0.010[O B S] \pm 0.005[G A L]) \times$ $10^{-12}$ and that gives an agreement

$$
\frac{\dot{P}_{b}^{G R}}{\dot{P}_{b}^{O B S}}=1.0023 \pm 0.0041[O B S] \pm 0.021[G A L]
$$

This is impressive and any deviation from general relativity due to any other source of energy loss must be at most $\sim O(0.1-1) \%$ of the general relativity predictions.

Most gravity theories alternative to general relativity, in particular scalar-tensor theories, predict the existence of dipole gravitational radiation as well [24, 20]. In a binary system the magnitude of this effect depends on the self-gravitational binding energies of the two bodies. Following always ref. [20], the predicted energy loss rate can be written

$$
\left.\frac{d E}{d t}\right|_{D}=-\frac{1}{3} \kappa_{D}<\frac{\mu^{2} m^{2}}{r^{4}} \mathcal{E}^{2}>
$$

where $\kappa_{D}$ depends on the theory in question and $\mathcal{E}$ is the difference in the self-gravitational binding energies per unit mass between the two bodies of the binary system. Expression (36) gives then

$$
\begin{aligned}
\left.\frac{d E}{d t}\right|_{D} & =-\frac{1}{3} \kappa_{D} \mathcal{E}^{2} \frac{\mu^{2} m^{2}}{\alpha^{4}} G(e) \\
\left.\frac{\dot{P}_{b}}{P_{b}}\right|_{D} & =-\kappa_{D} \mathcal{E}^{2} \frac{\mu m}{\alpha^{3}} G(e)
\end{aligned}
$$

where now

$$
G(e)=\left(1+\frac{1}{2} e^{2}\right)\left(1-e^{2}\right)^{-5 / 2}
$$

In the string theory under discussion, dipole terms come from the source term $\Sigma$ in the field equation (13) of the massless dilaton. Equivalently, it is the term $\mathcal{L}_{\text {int }} \sim \alpha\left(\phi_{0}\right)(\phi-$ $\left.\phi_{m}\right) \bar{\psi}_{n} \psi_{n}$ in the effective lagrangian for the dilaton interaction with the nucleon field $\psi_{n}$ (as applied to the macroscopic system of a binary pulsar) that leads to dipole radiation. For our 
purposes it suffices to consider precisely this dipole radiation effect. Either way, we find the expressions

$$
\begin{aligned}
\left.\frac{d E}{d t}\right|_{D} & \simeq-\frac{4 \alpha^{2}\left(\phi_{0}\right)}{3}\left(\frac{\Omega_{1}}{m_{1}}-\frac{\Omega_{2}}{m_{2}}\right)^{2} \frac{\mu^{2} m^{2}}{\alpha^{4}} G(e) \\
\left.\frac{\dot{P}_{b}}{P_{b}}\right|_{D} & \simeq-4 \alpha^{2}\left(\phi_{0}\right)\left(\frac{\Omega_{1}}{m_{1}}-\frac{\Omega_{2}}{m_{2}}\right)^{2} \frac{\mu m}{\alpha^{3}} G(e)
\end{aligned}
$$

where $\Omega_{1}, \Omega_{2}$ are the self-gravitational binding energies of the two bodies. Pf We now demand that the contributions (40)-(41) do not upset the agreement (35) of general relativity with the observed data for the binary pulsar PSR $1913+16$. This means that these contributions must be within $(0.1-1) \%$ of the corresponding contributions (32)-(33)as applied in general relativity. So, upon substituting the appropriate numerical values [20], we find for $\Delta \phi_{B P} \equiv$ $\left(\phi_{0}-\phi_{m}\right)_{B P}$ the bound

$$
\Delta \phi_{B P} \lesssim 10^{-(4-5)}
$$

of the same order of magnitude as the bound (29). So, both bounds from primordial nucleosynthesis and the binary pulsar essentially coincide with each other and with the attraction factor of the matter-dominated era as found in ref. [2].

\section{Inflation}

Since inflation [25] is a mechanism available in the early universe with which large exponential enhancements or supressions are associated, it would be interesting to see what is the effect of an inflationary epoch on the relaxation shift of the dilaton. In fact, it is not difficult to see that inflation could provide a very efficient early relaxation shift for the dilaton of an order well below all the limits already discussed. Suppose that the scalar field $\sigma$ in the action (6) plays the role of the inflaton and the potential $V(\sigma)$ satisfies the appropriate conditions

\footnotetext{
${ }^{2}$ Expressions (40)-(41) for the dipole radiation are only approximate. Formula (41), for example, should be corrected by the factors $\left[1+\alpha_{1}(\phi) \alpha_{2}(\phi)\right]\left[1+\frac{\beta_{A}}{1+\alpha^{2}\left(\phi_{0}\right)}\right]^{2}$. Here, the first factor comes from Kepler's third law, where the gravitational coupling is in fact an effective gravitational constant between the two self-gravitating stars with $\alpha_{1}(\phi), \alpha_{2}(\phi)$ strong-field modified values of $\alpha(\phi)$ inside the pulsar and the its companion. The second factor is due to the fact that the violation of the strong equivalence principle is proportional to the PPN parameter $\eta\left(=4 \beta_{E d d}-\gamma_{E d d}-3\right)$. We thank G.Esposito-Farese for bringing this point to our attention.
} 
for inflation. Then, in the quadratic approximation (17) for $B(\phi)$ with $\Delta \phi=\left(\phi-\phi_{m}\right)$, the field equation of $\phi$ gives ( upon neglecting the spatial variations )

$$
\Delta \ddot{\phi}+3 H \Delta \dot{\phi}+\frac{3}{2} H^{2} \kappa \Delta \phi=0
$$

where $H^{2} \simeq 2 q V(\sigma) / 3$ is the approximately constant Hubble parameter during the slow-roll period of inflation. Equation (44) is easily solved approximately by

$$
\Delta \phi \simeq e^{\left(-\frac{3}{2} \pm \sqrt{\frac{9}{4}-\frac{3}{2} \kappa}\right) \int H(t) d t} \simeq e^{-c H \tau}
$$

where $\mathrm{c}$ is some number $\sim O(1)$ and $\tau$ the period of inflation. So, for an inflationary epoch satisfying the usual duration condition $H \tau \gtrsim 70$ we expect a quick relaxation shift of the order

$$
\Delta \phi_{I N F} \lesssim 10^{-30}
$$

We see that, under some assumptions, inflation seems to be an extremely effective mechanism driving the dilaton field $\phi$ very early and in a quick and efficient way toward its equilibrium value $\phi_{m}$. If we then take into account the total attraction factor found in ref. [2], we see that today we expect an overall attraction factor ( due to inflation and the subsequent radiationand matter-dominated periods) of the order

$$
\Delta \phi_{0} \simeq 10^{-40}
$$

If this is true, it is evident that any deviations from general relativity would be extremely suppressed beyond any potentially observational limit.

A careful in depth analysis of inflation within the mechanism of ref. [2] has appeared very recently [26]. The authors of ref. 26] also study the quantum creation of dilatons during the primordial inflationary era and find that the resulting quantum fluctuations are naturally compatible with observational limits.

\section{Discussion}

The "least coupling" scheme introduced in ref. [2] for driving the dilaton vacuum expectation value toward values, which extremize the strengths of its interactions, is a very appealing 
proposal. The initial work of ref. [2] has studied the cosmological evolution of the dilaton field during the radiation- and matter-dominated eras of classical cosmology and showed that it can be safely reconciled with cosmological data. In the present work we have studied some additional cosmological and astrophysical constraints for the shift of the dilaton toward its equilibrium value in order to shed some light on how much close to that it has been settled down by today. Apart from the big-bang nucleosynthesis constraint (29), we have found a similar bound (42) for such a shift derived from the emission of massless dilatons from the binary pulsar. We have also seen that an inflationary era can easily drive the dilaton very quickly to values safely below these limits and the overall atraction factor of ref. [2]. In fact, if inflation is operative, the dilaton is driven to values which render the present scheme almost indistinguishable from general relativity. An in depth study of inflation within the mechanism of ref. [2] has been done in ref. 26]. 3]

From the fundamental theory point of view, the present scheme relies on some crucial assumptions. The basic assumption is that the various dilaton coupling functions $B_{i}(\Phi)$ admit a local maximum at some common value $\Phi_{m}$. This is guaranteed if all $B_{i}(\Phi)$ coincide with a common function $B(\Phi)$, factorized as in (1) and admitting a local maximum due to string loop effects as in (2). The crucial role here is played by the symmetries of the underlying string theory. The behaviour of the dilaton proper is related to the conjectured S-duality symmetry of the string theory. On the other hand, the T-duality symmetry, known to hold for some of the moduli fields, can be invoked to guarantee that the same scheme is also applicable to them. The character of these symmetries is lately a fruitful area of research and their relation to the scheme under discussion remains to be seen. f

\footnotetext{
${ }^{3}$ In another development, general relativity has been also shown to be an attractor of an arbitrary scalartensor theory in the context of stochastic inflation [27.

${ }^{4}$ It is indicative to mention here the following. We have assumed that inflation is driven by some field other than the dilaton ( or moduli in general [26]; recent alternative schemes include dilaton inflation [28], moduli inflation [29] and non-critical string theory inflation [30]). Supersymmetry is generally broken by the non-vanishing vacuum energy density present during inflation, thus lifting the flat directions of the effective supergravity theory. Flat directions are important for other reasons as well, e.g. for baryogenesis mechanisms. It is then reassuring that a "Heisenberg symmetry" can be used in the effective supergravity models to show that flat directions can be preserved [31]. The no-scale supergravity models, for which e.g. formula (11) is applicable, are a special case of these models. This fact further underlines the significance of some symmetries for the kind of theories we are considering.
} 
Our results, more specifically the bound (42) from the binary pulsar, were derived under the assumption that dilatons are massless. However, once realized that inflation, if present, is an extremely efficient way responsible for relaxing the dilaton toward its present value, one could possibly start speculating about possible values for the masses of the relevant scalar fields, namely the dilaton as well as the various moduli. In fact, the authors of ref.[26] remark that a mass term is possible and does not create the usual Polonyi [32]- moduli [4, 33] problem ( associated with particles of mass $\sim O\left(M_{W}\right)$ and gravitational strength couplings to ordinary matter, which dominate the energy density of the universe until the temperature is too low for nucleosynthesis to occur). This is simply achieved here because, after inflation, the vacuum expectation value of the relevant field is left very precisely at the place where it stores no potential energy. Moreover, the same authors find that there is a very wide range of masses exceeding $\sim 10 \mathrm{GeV}$ and extended up to the Planck scale $M_{P}$, for which dilatons or moduli are essentially stable and dominate the mass density of the universe, offering thus the possibility of a novel type of dark matter.

However, there is no apparent reason to treat the dilaton on the same footing as the other moduli. The dilaton is intimately connected with the graviton and its vacuum expectation value determines the gauge coupling constant at the string scale, whereas the moduli are associated with compactified dimensions and their real part determines the radii of them. There are cases with massless dilatons and massive moduli. Moreover, some arguments have been put forward [33, 34] that a mechanism for cancelation of the cosmological constant requires a light weakly scalar field - the dilaton - with a mass about the fourth root of the observational bound of the cosmological constant-vacuum energy $\left(10^{-46} \mathrm{GeV}^{4}\right)^{1 / 4} \sim 10^{-3} \mathrm{eV}$. Depending on the mechanism transmitting the supersymmetry breaking to the standard model, there is the possibility of associating a light dilaton $m_{\phi} \sim m_{S U S Y}{ }^{2} / M_{P}$ of a mass of this order of magnitude ( corresponding to a supersymmatry breaking scale $\sim(1-10) \mathrm{TeV}$ ) with a non-vanishing cosmological constant. In fact, there is a diverse set of recent observations suggesting that the universe may possess a non-zero cosmological constant 35. We still need inflation to avoid overclosure of the universe in the present epoch [26]. The possibility, however, of a light dilaton, associated with an interconnection between a small vacuum energy of order e.g. $\exp \left(-c 4 \pi / g_{e m}{ }^{2}\right) M_{P}{ }^{4}$ ( compare with the structure of formulae 
(9), (11) ) and a not completely exact symmetry responsible for diminishing the cosmological constant and related to the present scheme, remains as an intriguing one. 


\section{Acknowledgements}

I acknowledge informative discussions with C.Kounnas, a stimulating talk by J.H.Taylor

and the kind hospitality of CERN Theory Division. I am also indebted to G.Esposito-Farese for valuable correspondence concerning the interpretation of the binary pulsar data. 


\section{References}

[1] J.Ellis, S.Kalara, K.A.Olive and C.Wetterich, Phys.Lett.B228 (1989) 264.

[2] T.Damour and A.M.Polyakov, Nucl.Phys.B423 (1994) 532.

[3] M.Gasperini and G.Veneziano, Phys.Rev.D50 (1994) 2519; M.Gasperini, Phys.Lett. B327 (1994) 214.

[4] B.de Carlos, J.A.Casas, F.Quevedo and E.Roulet, Phys.Lett. B318 (1993) 447; T.Banks, D.B.Kaplan and A.Nelson, Phys.Rev. D49 (1994) 779.

[5] T.Damour and A.M.Polyakov, Gen.Rel.Grav. 26 (1994) 1171.

[6] T.Damour and K.Nordtvedt, Phys.Rev.Lett. 70 (1993) 2217; Phys.Rev. D48 (1993) 3436 .

[7] E.S.Fradkin and A.A.Tseytlin, Phys.Lett. B158 (1985) 316; C.G.Kallan, D.Friedan, E.J.Martinec and M.J.Perry, Nucl.Phys. B262 (1985) 593.

[8] A.Font, L.Ibanez, D.Lüst and F.Quevedo, Phys.Lett. B249 (1990) 35; S.J.Rey, Phys.Rev. D43 (1991) 526; S.Kalara and D.V.Nanopoulos, Phys.Lett.B267 (1991) 343; J.H.Schwartz and A.Sen, Phys.Lett. B312 (1993) 105; Nucl.Phys. B411 (1994) 35; C.Vafa and E.Witten, Nucl.Phys. B431 (1994) 3.

[9] A.Giveon, M.Porrati and E.Rabinovici, Phys.Rept.244 (1994) 77; E.Kiritsis and C.Kounnas, CERN-TH.7328/94, hep-th/9407005, Proceedings of the 2-eme Journee Cosmologie, Observatoire de Paris, 2-4 June 1994 (World Scientific, eds. H.de Vega and N.Sanchez) and references therein.

[10] A.Sen, Int.J.Mod.Phys. A9 (1994) 3707; J.H.Schwartz, CALT-68-1965, hep-th/9411178; CALT-68-1984, hep-th/9503127 and references therein.

[11] I.Bakas, Phys.Lett. B343 (1995) 103; C.Hull and P.Townsend, QMW-94-30, hepth/9410167. 
[12] V.Kaplunovsky, Nucl.Phys. B307 (1988) 145; ibid B382 (1992) 436 (E).

[13] E.Cremmer, S.Ferrara, C.Kounnas and D.V.Nanopoulos, Phys.Lett. 133B (1983) 61; J.Ellis, A.B.Lahanas, D.V.Nanopoulos and K.Tamvakis, Phys.Lett. 134B (1984) 429; J.Ellis, C.Kounnas and D.V.Nanopoulos, Nucl.Phys. B241 (1984) 373; ibid B247 (1984) 373; Phys.Lett. 143B (1984) 410; for a review, see A.B.Lahanas and D.V.Nanopoulos, Phys.Rept. 145 (1987) 1; see also P.Binetruy, S.Dawson, M.K.Gaillard and I.Hinchliffe, Phys.Rev. D37 (1988) 2633.

[14] T.P.Walker, G.Steigman, D.N.Schramm, K.A.Olive and K.Kang, Ap.J. 376 (1991) 51.

[15] G.Steigman, OSU-TA-22/94(1994); K.A.Olive and G.Steigman, Ap.J.Supp.(1995) in press; C.J.Copi, D.N.Schramm and M.S.Turner, Science 267(1995) 192; L.M.Krauss, CWRU-P1-95 ( Nucl.Phys.B, Proceedings and Supplements, to appear); S.Sarkar, OUTP-95-16P, hep-ph/9602260, Rep.Prog.Phys. in press.

[16] E.W.Kolb, M.J.Perry and T.P.Walker, Phys.Rev. D33 (1986) 869; J.D.Barrow, Phys.Rev. D35 (1987) 1805; V.V.Dixit and M.Sher, Phys.Rev. D37 (1988) 1097; J.A.Casas, J.Garcia-Bellido and M.Quiros, Mod.Phys.Lett. A7 (1992) 447; Clas.Quant.Grav. 9 (1992) 1371; R.J.Scherrer and D.N.Spergel, Phys.Rev. D47 (1993) 4774; B.A.Campbell and K.A.Olive, Phys.Lett. B345 (1995) 429.

[17] J.Casser and H.Leutwyler, Phys.Rept. 87 (1982) 77; H.Leutwyler, BUTP-94/8 (1994).

[18] R.D.Blandford et al. eds., Pulsars as physics laboratories, Phil.Trans.R.Soc.London A341 (1992) 1.

[19] E.S.Phinney and S.R.Kulkarni, Ann.Rev.Astr.Astroph. 32 (1994) 591.

[20] C.M.Will, Theory and Experiment in Gravitational Physics, revised ed. (Cambridge Univ. Press, 1993); Int.J.Mod.Phys. D1 (1992) 13.

[21] T.Damour, Gravitation and Experiment in Gravitation and Quantizations, eds. B.Julia and J.Zinn-Justin, Les Houches, Session LVII (Elsevier, 1994); see also gr-qc/9412064, 9412074 . 
[22] R.A.Hulse and J.H.Taylor, Ap.J.(Letters)195 (1975) L51; see also the 1993 Nobel lectures in physics: R.A.Hulse, Rev.Mod.Phys. 3 (1994) 699; J.H.Taylor, ibid 711.

[23] P.C.Peters and J.Mathews, Phys.Rev. 131 (1963) 435.

[24] C.M.Will and H.W.Zaglauer, Ap.J. 346 (1989) 366.

[25] A.H.Guth, Phys.Rev. D23 (1981) 347; for a review, see A.D.Linde, Particle Physics and Inflationary Cosmology (Harwood, 1990); E.W.Kolb and M.S.Turner, The Early Universe (Addison-Wesley, 1990); K.A.Olive, Phys.Rept. 190 (1990) 307.

[26] T.Damour and A.Vilenkin, hep-th/9503149, Phys.Rev. D in press.

[27] J.Garcia-Bellido and D.Wands, SUSSEX-AST-95/3-1, gr-qc/9503049.

[28] G.Veneziano, Phys.Lett. B265 (1991) 287; M.Gasperini and G.Veneziano, Astropart. Phys. 1 (1993) 317.

[29] T.Banks, M.Berkooz, G.Moore, S.H.Shenker and P.J.Steinhardt, hep-th/9503114, Phys.Rev. D52 (1995) 3548; S.Thomas, SLAC-PUB-95-6767, hep-th/9503113.

[30] J.Ellis, N.Mavromatos and D.V.Nanopoulos, hep-th/9503162.

[31] M.K.Gaillard, H.Murayama and K.A.Olive, hep-ph/9504307, Phys.Lett. B355 (1995) 71.

[32] G.D.Coughlan, W.Fischler, E.W.Kolb, S.Raby and G.G.Ross, Phys.Lett. B131 (1983) 59; J.Ellis, D.V.Nanopoulos and M.Quiros, Phys.Lett. B174 (1986) 176; O.Bertolami, Phys.Lett. B209 (1988) 277.

[33] T.Banks, M.Berkooz and P.J.Steinhardt, hep-th/9501053, Phys. Rev. D52 (1995) 705.

[34] L.Susskind (unpublished), see ref.[33]; see also S.R.Beane, DUKE-TH-95-84.

[35] L.M.Krauss and M.S.Turner, astro-ph/9504003, Gen. Rel. Grav. 27 (1995) 1137; J.P.Ostriker and P.J.Steinhardt, astro-ph/9505066. 